\title{
Cognitive components of reaction time in Parkinson's disease
}

\author{
Nigel Jordan, Harvey J Sagar, James A Cooper
}

\begin{abstract}
Studies of reaction time in Parkinson's disease (PD) have suggested a selective deficit in simple reaction time (SRT), compared with choice reaction time (CRT). This finding has been interpreted as a deficit in motor preprogramming but could involve other factors, such as attentional focussing and stimulus predictability. Moreover, not all studies show the same selective deficit, possibly because of differences in patient selection and treatment effects. The neurochemical basis of $R T$ deficits in $P D$ remains unclear. Accordingly, the contribution of cognitive factors to impaired RT was assessed in a large group of $P D$ patients, including early untreated cases, and performance was examined in relation to clinical variables and the effect of treatment in longitudinal study. Motor output was constant in both SRT and CRT tasks. In the SRT task, all stimuli required a response; in the CRT task, subjects were required to respond to only one of the two possible stimuli. Attentional focussing on SRT was examined by variation of the interval between cue and stimulus; effects of stimulus uncertainty were evaluated from a comparison of SRT and CRT; temporal predictability of the stimulus was examined from a comparison of conditions in which the interval between warning signal and imperative stimulus was constant or variable. The PD patients showed similar deficits in SRT and CRT, but normal effects of cue-stimulus interval and temporal predictability. Reaction time correlated with measures of global cognitive capacity and frontal-lobe function, as well as motor disability. Treatment had no effect on SRT or CRT, despite clinical benefit. These findings indicate that RT deficits in PD are not due to impaired attentional focussing or stimulus predictability but are compatible with a deficit in higher-order processes concerned with the orientation of both cognitive and motor responses to a stimulus. These processes are not substantially dopaminedependent but may be served by nondopaminergic neurotransmission.
\end{abstract}

(F Neurol Neurosurg Psychiatry 1992;55:658-664)

Motor deficits, notably akinesia, are universal clinical features of Parkinson's disease. Howev- er, a number of cognitive deficits have also been recognised involving attention, memory, frontal lobe function, conceptual ability and visuospatial function. ${ }^{12}$ The relationship between the cognitive and motor disturbances is unclear, but deficits in "higher order" motor control, such as motor sequencing, ${ }^{34}$ double simultaneous movements ${ }^{5}$ and the learning of new motor skills ${ }^{6}$ have been recognised in PD and may be based more on cognitive dysfunction than pure motor impairment.

Formal explorations of the role of cognitive factors in the initiation of movement are scanty. Results of experiments on motor reaction time, however, have been used to support the concept of a deficit in the "preprogramming" of movement in PD. Specifically, PD patients have been claimed to show prolongation of simple reaction time (SRT), in which subjects respond in the same way to all stimuli regardless of nature, but not choice reaction time (CRT) where a different response is required depending on the stimulus. Thus, for example, a difference between SRT and CRT of approximately 100 to $200 \mathrm{~ms}$ in normal elderly subjects ${ }^{7}$ contrasts with a difference of only $50 \mathrm{~ms}$ in patients with PD. ${ }^{89}$ The advantage gained by normal subjects in SRT compared with CRT has been attributed to the ability to make advanced preparation of the movement in SRT but not CRT; thus the inability of PD patients to benefit in SRT compared to CRT has been ascribed to a deficit in this motor preprogramming. Other factors, however, could explain the pattern of results on SRT and CRT in PD. In particular, attentional factors would be expected to be less important in CRT, where no advance preparation is possible, than in SRT where the same preparation is required for all responses. For example, the response required from an awaited stimulus is uncertain in CRT but not in SRT so that attentional focussing may contribute less to efficient performance in CRT compared to SRT. In normal subjects, RT is dependent on the interval between a warning signal and the imperative stimulus that tells the subject to move; with increasing warning intervals, RT becomes faster. These observations indicate that response preparation is involved in the movement tapped by the reaction time tasks. In PD, some studies have shown that reaction time does not decrease with increasing warning intervals in the way that it does in normal subjects and other studies have demonstrated an effect of attentional manipulation on the SRT deficits of PD. ${ }^{10}$ These observations suggest that part of 
Table 1 Characteristics of subject groups. Figures given as means and, in brackets, standard error of the mean

\begin{tabular}{lllllll}
\hline Group & M:F & Age & $\begin{array}{l}\text { Disease } \\
\text { Duration } \\
\text { (years) }\end{array}$ & $\begin{array}{l}\text { Education } \\
\text { (years) }\end{array}$ & $\begin{array}{l}\text { NART } \\
\text { IQ }\end{array}$ & $\begin{array}{l}\text { KCRS } \\
\text { score }\end{array}$ \\
\hline Healthy Control & $13: 11$ & $58 \cdot 6(4 \cdot 2)$ & - & $10 \cdot 3(0 \cdot 7)$ & $108(6 \cdot 7)$ & $\overline{15}$ \\
Parkinson Untreated & $18: 14$ & $57 \cdot 7(3 \cdot 6)$ & $1 \cdot 6(0 \cdot 2)$ & $9 \cdot 4(0 \cdot 5)$ & $102(6 \cdot 0)$ & $15 \cdot 3)$ \\
Parkinson Treated & $20: 14$ & $59 \cdot 9(3 \cdot 1)$ & $4 \cdot 1(2 \cdot 3)$ & $9 \cdot 9(0 \cdot 6)$ & $110(6 \cdot 6)$ & $19 \cdot 0(2 \cdot 0)$ \\
\hline
\end{tabular}

the deficit in SRT in PD may arise from cognitive factors involved in attentional focussing, stimulus prediction or response selection.

In addition to these difficulties of interpretation, the pattern of results is not consistent across studies as some authors have found equal prolongation of SRT and CRT in PD. ${ }^{11}$ The reasons for these discrepancies are unclear but may be related to other clinical variables that influence reaction time but which have not been accounted for in the experimental design and which differ between studies. Thus, for example, cognitive impairment may influence reaction time independently of motor control; depression may prolong reaction time through its effect of producing psychomotor retardation; disease chronicity and the nature and duration of treatment may influence SRT and CRT differentially. ${ }^{12}$ Moreover, the varied nature of the tasks employed in previous reports may account for some of the differences described.

This study examined SRT in PD; CRT was also examined using a go/no-go paradigm in which motor response was constant; response was manipulated only by altering the instructions to the subject. Thus because motor preprogramming is possible for all responses, we did not address the question of the extent to which PD patients select and execute a motor programme (Marsden 1982). This study is particularly concerned with the role of cognitive factors in the origin of impaired SRT in PD and considers the following questions: (1) Is slowed response execution of PD patients in an externally cued SRT task affected by nonspecific response preparation (attentional focussing)? This was addressed from two components of the design: First, by manipulation of the interval between warning signal and imperative stimulus; and, second, from the effects of stimulus uncertainty as shown by a comparison of conditions in which all stimuli require a response (SRT) and those in which only some stimuli require a response (CRT).

(2) Is slowed response execution of PD patients in an externally cued SRT task affected by poor temporal predictability of the stimulus? Temporal predictability is addressed by comparing the effects of SRT conditions with a fixed interval between warning signal and imperative stimulus, which the subjects can learn to predict, and those in which the interval was randomly varied.

(3) Is slowed response execution of PD patients in an externally cued SRT task correlated with cognitive deficits and depression? (4) Are any of these factors improved by drug treatment? This question is addressed by comparing patients on and off medication and by longitudinal study of patients before and after treatment.

\section{Methods \\ Subjects}

The subject groups comprised 32 patients with newly-diagnosed, untreated $\mathrm{PD}, 34$ patients with PD currently being treated and 24 healthy control subjects (HCS) (table 1). Newlydiagnosed PD patients were drawn from consecutive referrals to the Department of Neurology. The diagnosis was based on the presence of akinesia plus rigidity, rest tremor or postural instability and absence of clinical signs of other causes of Parkinsonism. Treated PD patients were willing participants drawn from the outpatient clinics of the Neurology Department. The subject groups did not differ significantly in age, sex or IQ. The untreated and treated PD subgroups differed significantly in disease duration but not in clinical motor disability. Thus more advanced disease in the treated PD group was masked by treatment so that, for the purposes of this experiment, the groups were matched in clinical motor disability. Medication in the treated group comprised a single drug in 31 patients: a levodopa preparation (14 patients, mean dose $200 \mathrm{mg} /$ day, range 100 to $500 \mathrm{mg} /$ day), bromocriptine (11 patients, mean dose $15 \mathrm{mg} /$ day, range 4 to $35 \mathrm{mg} /$ day), or benzhexol (6 patients, mean dose $8 \mathrm{mg} /$ day, range 2 to $30 \mathrm{mg} /$ day). Three patients were receiving polypharmacy, usually a levodopa preparation plus an anticholinergic drug.

Twenty one of the untreated patients were reassessed after randomisation to monotherapy of levodopa, bromocriptine or benzhexol. Although some individual patients in the benzhexol or bromocriptine-treated groups showed a weak clinical response, treatment produced a significant improvement in clinical motor disability in the total group, as measured by score on the King's College Rating Scale (KCRS) $(p<0.01)$. Fourteen of the control subjects were reassessed at a similar interval.

None of the subjects had a past history of head injury, alcohol abuse or other neurological or general medical condition that might produce motor or cognitive impairment. No subject was receiving psychoactive medication. None of the untreated patients had received levodopa or bromocriptine at any time before the study.

\section{Procedure}

The memory and orientation section of the Blessed Dementia Scale (BDS) ${ }^{13}$ was used to quantify the degree of overall cognitive impairment. Intelligence quotient (IQ) was estimated using the National Adult Reading Test. ${ }^{14}$ Memory was assessed using the Wechsler Memory Scale ${ }^{15}$ which provides a memory quotient (MQ) parallel to IQ in intelligence. Frontal lobe function was assessed from the number of categories, number of perseverative responses and number of cards to first category on the Wisconsin Card Sorting Test (WCST). ${ }^{16}$ Affective disturbance was quanti- 
fied by the Beck Depression Inventory (BDI). ${ }^{17}$ Overall motor disability was assessed using the Kings College Rating Scale (KCRS), a detailed quantitative measure of clinical symptoms and signs. ${ }^{18}$ Finger dexterity (Fine Finger Movements; FFM) was evaluated from the ability of subjects to rotate a spindle between the thumb and forefinger of each hand; results were expressed as the number of revolutions achieved in thirty seconds, averaged across the two hands.

\section{Simple reaction time (SRT)}

(1) Standard cue-stimulus interval During the experiment, the subject sat facing a VDU monitor, positioned at eye level. The hand to be tested was supported by an arm rest fixed to the chair so that the index finger could be comfortably placed on the response button. SRT was assessed using a BBC Master Series microcomputer and high resolution VDU monitor. Subjects received an auditory warning signal of $250 \mathrm{~ms}$ duration, one second before the imperative stimulus, a yellow coloured square of $25 \mathrm{~cm}^{2}$ area, which appeared centrally on the screen. Subjects were required to respond to the appearance of the stimulus as rapidly as possible with a single press of the space carriage bar of the computer keyboard. The subjects were given standardised instructions and allowed a practice trial of ten responses to achieve asymptotic improvement in performance. Trials proper required twenty responses from each hand. Both hands were tested, separately and in random order. The computer measured responses within an accuracy of ten ms. Results are expressed as the mean of the responses from the two hands.

(2) Variable cue-stimulus interval In a second experimental condition, the interval between warning signal and imperative stimulus was varied. As in the first condition, an auditory tone of $250 \mathrm{~ms}$ duration was presented before the appearance of the stimulus, a $25 \mathrm{~cm}^{2}$ yellow square, on the VDU screen. In this second condition, however, the interval between the warning signal and the appearance of the stimulus was varied in $100 \mathrm{~ms}$ steps between 100 and $1500 \mathrm{~ms}$, presented in random order. Five responses were required at each of the fifteen intervals to give a total of seventy-five responses for each hand. Patients were given standardised instructions and allowed ten practice responses. Results are expressed as the mean SRT for each of the 15 cue-stimulus intervals averaged across both hands.

Choice reaction time (CRT) As in the standard SRT condition, subjects received an auditory warning signal of $250 \mathrm{~ms}$ duration, one second before the presentation of the stimulus. For CRT, however, the stimuli were of two different types, yellow and purple squares each of $25 \mathrm{~cm}^{2}$. Stimuli were presented in random order. Subjects were required to respond to the yellow square throughout to minimise the memory demands of the task. Forty responses (twenty correct responses) were carried out for each hand, and results were calculated as the mean of all correct responses.

Statistical analysis Differences between data sets were evaluated by repeated measures analysis of variance (ANOVA). Paired comparisons were made using unpaired or paired Student's $t$ test (two-tailed, except where indicated otherwise). Correlations between variables were assessed using Pearson's correlation coefficient.

\section{Results}

\section{Effects of temporal predictability}

Effects of temporal predictability were assessed from a comparison of the SRT condition in which the interval between the warning signal and the imperative stimulus was constant and that in which it varied randomly. A repeated measures ANOVA of Group (treated PD, untreated PD, HCS) by Condition (fixed interval, variable interval) revealed main effects of Group $(p<0.001)$ and Condition $(p=0.005)$ but no interaction $(p=0.75)$ Planned paired comparisons showed that the two PD groups were impaired on both tasks, relative to healthy control subjects $(p<0.001)$ but did not differ from each other. The main effect of Condition was accounted for by an improvement in RT when the stimulus became predictable (fixed interval condition).

Treatment had no effect on temporal predictability as shown by a repeated measures ANOVA of Group by Condition for the two PD subgroups only. The main effect of Condition was again significant $(p<0.01)$ but the effect of Group and the Group by Condition interaction were not significant.

Subsequent analyses assessed the effect of stimulus uncertainty and attentional focussing on RT.

\section{Fixed cue-stimulus interval (fig 1)}

Effect of stimulus uncertainty was assessed by a repeated measures ANOVA of Group (treated PD, untreated PD, HCS) by Condition (SRT, CRT). The analysis showed main effects of Group $(p=0.001)$ and Condition

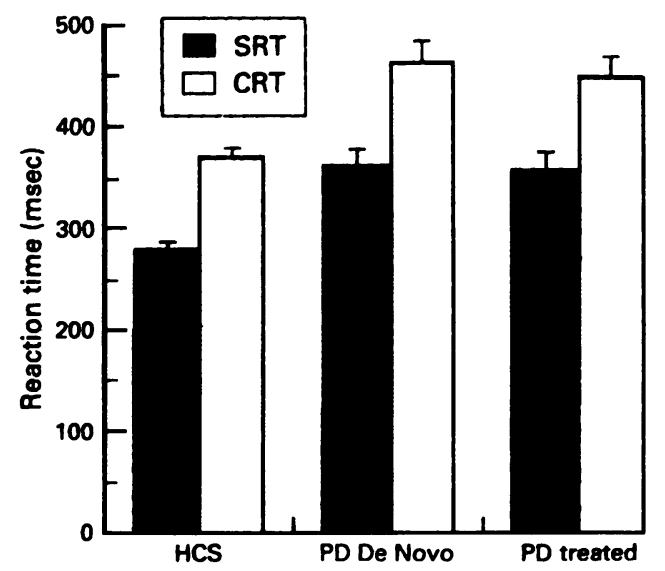

Figure 1 Simple and choice reaction time in fixed cue-stimulus condition (mean and SEM). De novo and treated PD patients showed prolonged simple and choice reaction time but the groups did not differ from each other 
Figure 2 Simple reaction cue-stimulus condition (mean and SEM). De novo and treated $P D$ patients showed prolonged $S R T$ at all intervals but did not differ from normal subjects in the effect of interval on $S R T$ time in variable

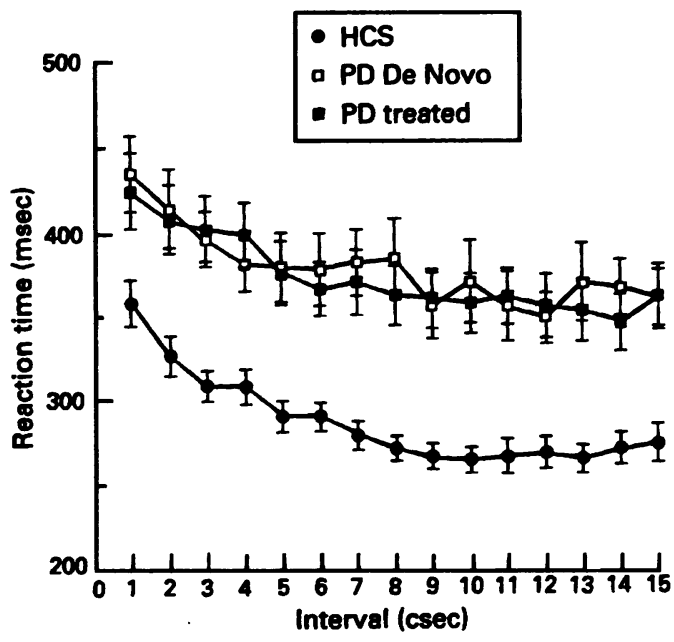

( $p<0.00001)$ but no interaction $(p=0.85)$. Planned paired comparisons showed that both the newly diagnosed, untreated patients and those already taking medication were slower than the normal control subjects $(p<0.01)$ but the two PD subgroups did not differ significantly from each other. Similar analyses were carried out for SRT and CRT separately. Results showed that untreated patients were slower than the controls in both SRT $(p<0.0005)$ and CRT $(p<0.002)$, as were the treated patients $(p=0.001, p<0.005$ respectively); the two $P D$ subgroups did not differ from each other in either condition $(p=0.9, p=0.7)$.

Variable cue stimulus interval (fig 2)

Attentional focussing was assessed by a repeated measures ANOVA of Group by Condition (cue-stimulus interval). The analysis showed main effects of Group ( $p<0.001)$ and Condi-

Figure $3 \quad S R T$ and $C R T$ (mean and $S E M)$ before (1) and after (2) treatment in de novo $P D$ compared with normal subjects assessed on two occasions, separated by a similar interval. Treatment had no effect on response.
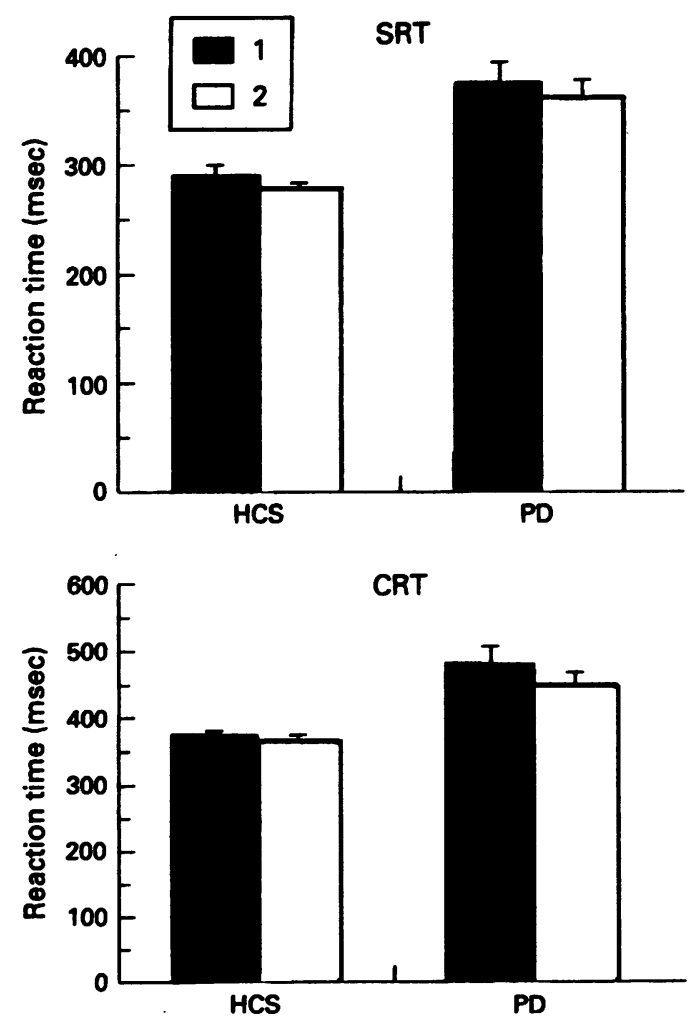

tion ( $p<0.0001$ ) but no Group by Condition interaction. Thus although slower overall, both PD subgroups benefitted normally from the advance information provided by the cue. Planned paired comparisons showed that both PD subgroups, untreated and treated, differed from the control group $(p<0.01)$ but the two PD subgroups did not differ significantly from each other.

Effect of treatment in longitudinal study (fig 3)

For patients tested before and after treatment, repeated measures ANOVA of Group by Condition (SRT, CRT) by Assessment (first and second assessments for control group; before and after treatment for PD group) showed main effects of Group ( $p<0.002)$ and Condition $(p<0.0001)$ but no interactions of Condition by Assessment $(p=0.32)$, Group by Condition ( $p=0.55)$ or Group by Assessment $(p=0.56)$. The lack of significant interactions indicates that treatment did not improve simple or choice reaction time of the PD patients despite significant improvement in clinical motor disability. Moreover, the nature of the drug treatment did not affect performance in the PD group. Thus reaction time appears to be largely independent of treatment in PD.

Correlations of reaction-time with cognitive, affective and clinical motor disability (table 2) These correlations were performed on the total PD group because no significant differences were found between the two PD subgroups. Significant correlations were found between both simple and choice reaction time and clinical motor score as measured by the KCRS $(r=0.50, p=0.0001$ and $r=0.54, p<0.0001$ respectively) and FFM ( $r=0.61, p<0.0001$ and $r=0.55, p<0.0001$ respectively). Cognitive capacity as measured by the BDS correlated with both SRT and CRT ( $r=0.35$, $\mathrm{p}<0.01$ and $\mathrm{r}=0.30, \mathrm{p}<0.05$ respectively). In addition, number of perseverative responses correlated significantly with SRT ( $r=0.37$; $\mathrm{p}<0.01$ ) although not with CRT ( $r=0.04$; $\mathrm{p}=0.81$ ); no significant correlation was found between RT and the other cognitive measures (table 2). No correlations were found between either simple or choice reaction times and depression, as measured by the BDI ( $r=0 \cdot 25$, $p=0.27$ and $r=0.12, p=0.60$ respectively). Furthermore, comparison between those PD patients deemed depressed and those deemed non-depressed according to score on the BDI showed no significant differences for SRT $(p=0.35)$ or CRT $(p=0.44)$.

Table 2 Correlation coefficients $(r)$ between reaction time (SRT, CRT) and measures of cognitive, affective and clinical motor disability

\begin{tabular}{lrr}
\hline & \multicolumn{1}{c}{$S R T$} & \multicolumn{1}{c}{$C R T$} \\
\hline BDS & 0.35 & 0.30 \\
BDI & 0.25 & 0.12 \\
MQ & -0.23 & -0.12 \\
WCST & & \\
$\quad$ categories & -0.27 & 0.18 \\
$\quad$ perseverative responses & 0.37 & 0.04 \\
$\quad$ cards to 1st category & -0.09 & -0.11 \\
Fine finger movements & 0.61 & 0.55 \\
KCRS score & 0.50 & 0.54 \\
\hline
\end{tabular}




\section{Discussion}

This study examined the effects of attentional focussing, temporal predictability and stimulus uncertainty on SRT and go/no-go CRT in PD and related performance to other cognitive and affective measures and the effects of treatment. The results indicate that:

(1) The prolonged SRT in PD was not caused by lack of temporal predictability, stimulus uncertainty or poor attentional focussing.

(2) None of the factors examined in this RT study was affected by therapy sufficient to produce improvement in clinical motor disability.

(3) RT in PD was correlated with cognitive impairment.

\section{SRT-CRT differences in PD}

Results of studies of SRT and CRT in PD are controversial; some studies have shown a selective abnormality of SRT $^{8919}$ whereas others have shown deficits in both SRT and CRT. ${ }^{12} 20$ Reasons for these discrepancies are not clear but may relate to patient selection as well as experimental design.

"Choice" in a CRT experiment may be choice of movement or choice of correct stimulus. Most studies have used choice of movement as the response variable, including right or left index finger, 9 direction of movement in response to the appearance of a cursor, ${ }^{19}$ right or left movement, ${ }^{12}$ and right or left arm, direction and extent of movement. ${ }^{20}$ In our experiment, however, "choice" was one of correct stimulus, the motor response being constant. The critical difference between this and previous studies is that in our CRT experiment not all the stimuli required a movement whereas, in other studies, each stimulus required a movement, albeit of a different nature. Our CRT paradigm used a constant motor output; subjects were simply required to decide whether or not to respond. We cannot therefore address directly the issue of motor preprogramming deficits in PD. However, the finding of equivalently impaired performance on the SRT and CRT tasks suggests that the PD patients are not specifically affected by uncertainty as to whether or not the stimulus will appear; that is, slowness of response in PD is unlikely to be due to abnormal response criteria. In so far as these "confidence ratings" are a reflection of attentional control and executive processes, such as prediction, the results suggest that deficits in these domains are not influential at this simple level of response.

\section{Effects of attentional focussing}

We predicted that a deficit in attentional focussing in PD would lead to greater slowing of SRT at shorter cue-stimulus intervals but this result was not obtained. Indeed, the pattern of response across cue-stimulus intervals was qualitatively normal after the first $100 \mathrm{~ms}$. Normal effects of variable cue-stimulus interval have also been shown by Rafal $e t$ $a .^{21}$ Thus the results of the variable cuestimulus condition suggest that abnormal SRT in PD is either the simple result of motor slowing, is due to poor attentional focussing within the first $100 \mathrm{~ms}$ or is due to higher order cognitive deficits. The correlations between SRT, CRT and global cognitive capacity as assessed by the Blessed Dementia Scale, and between SRT and perseverative responses on the Wisconsin Card-Sorting Test, support a role of cognitive deficits in the origin of abnormal reaction time in PD. Surprisingly, depression was found to have no influence on reaction time in this experiment. Other observations from our laboratory, however, do support an effect of depression upon reaction time when assessed in an uncued paradigm. ${ }^{22}$ In general, the results suggest a substantial effect of cognitive factors in reaction time in PD but do not support specific deficits in attentional focussing, or stimulus prediction. Although Bloxham et $a^{23}$ showed differences in the effect of cue-stimulus interval on SRT of PD and control subjects, the major difference was at the longest interval $(3200 \mathrm{~ms})$ and the authors concluded that PD patients function in SRT as if performing a secondary task but allocate attentional resources normally. Our results are compatible with this interpretation. Of particular interest in our study is the finding of a significant correlation between SRT and number of perseverative responses on the Wisconsin Card-Sorting Test, a traditional measure of frontal-lobe function. Although no correlation was found with CRT, and interpretation must be tentative at this stage, this result does suggest that frontal-lobe function may be a critical influence on reaction time in PD and, by implication, in the genesis of normal movement.

\section{Brain-behaviour relationships in reaction time} tasks

The basal ganglia have been considered important in the preparation, selection and execution of learned motor programmes. ${ }^{24}$ In SRT, all stimuli require a response so that the movement can be prepared in advance of the stimulus; in CRT, by contrast, the required movement depends upon the nature of the stimulus, so that the movement cannot be prepared in advance. The finding in some studies of a selective deficit in SRT relative to CRT in PD has led to the suggestion that patients with $P D$ are impaired in motor preprogramming, that is, the ability to self-direct the preparation and selection of motor programmes in advance of an external stimulus to respond. $^{8919}$

SRT and CRT have been considered to depend upon separate routes for action. A "fast" route for SRT is dependent on attentional focussing; a direct route for CRT is slower and attentional focussing is not required. ${ }^{25}$ The fast route is largely controlled by internal control of attention whereas the direct route is largely automatic and controlled by external stimuli. The Bereitschaftspotential or "readiness" potential is detectable as a midline cortical potential immediately preceding voluntary movement and may arise from activation of the loop between the supplementary motor area (SMA), the basal ganglia and 
the ventrolateral thalamus; the SMA is considered to be involved in "motor readiness." 26 In PD, some studies indicate diminution in the amplitude of this potential ${ }^{27}$ implying disruption of links with the supplementary motor area as critical to the origin of disordered voluntary movement in PD. More recently positron emission tomography has demonstrated activation of the SMA when normal subjects carry out motor tasks dependent on internal cues (where movement preparation is possible in advance) and especially on tasks in which they generate a random selection between different movements. ${ }^{28}$ Preliminary observations in PD suggest impaired activation of the SMA during attempts to conduct these tasks (Passingham, personal communication). Together, these observations suggest that a fundamental deficit in disordered voluntary movement in PD arises from disruption of the basal ganglia-SMA connections which leads to a deficit in the execution of motor output through internal cues. With externally-cued movement, input from the sensory cortex feeds directly into premotor cortex, bypassing the SMA, so that PD patients perform these movements much better. ${ }^{29}$.

How can these conclusions be related to the results of our study? Factors that influenced motor readiness might be expected to have disproportionate effects in PD if they operated through the SMA but this prediction was not fulfilled and other explanations must be considered. First, the finding that RT in PD is not differentially influenced by attentional focussing, temporal predictability or stimulus uncertainty suggests that these attentional factors may be acting independently of the SMA and are acting through a direct route to the premotor cortex. Second, the lack of effect may be related to the experimental design. The warning signal acts as an external cue so that the effects of interval in this design may operate preferentially on the premotor cortex. Further studies should examine the effects of interstimulus interval in a continuous, uncued reaction time paradigm. Third, the PD patients may achieve normal effects of attentional manipulation in RT tasks by attentional overcompensation. PET studies, for example, have shown that cortical activation is inversely related to cognitive performance in a verbal fluency task, implying that poor performers show less efficient cortical metabolism. ${ }^{30}$ This hypothesis predicts that the attentional manipulations incorporated in the experiment reported here would be accompanied by supernormal activity in frontostriatal pathways in PD.

\section{Effects of treatment}

Several studies have considered the relationship between reaction time and activity in dopaminergic pathways, by observing the effect of medication or the changes during onoff swings. Rafal et $a l^{21}$ studied ten patients, four before and after treatment with levodopa and six during marked on-off swings. Analyses of reaction times were performed in terms of unremediated, that is, before treatment or "off" and remediated, that is, after treatment or "on". The unremediated patients had slower reaction times than the remediated patients. All cases, however, had advanced disease, the disease duration ranging from six to twenty years; the cognitive and affective state of the patients is unclear; the changes in clinical motor disability were marked; the results were derived from a mixed group of patients, including some with age of onset as young as 27 years and one who had undergone a thalamotomy; and treatment was variable among subjects. Similar problems hamper interpretation of other studies. Pullman et al ${ }^{12}$ examined five patients aged 29-61 years with Hoen and Yahr disability ratings of II to IV, who were not clinically depressed, before and after intravenous infusions of levodopa. The treatment switched the patients from an "off" to an "on" state. This change was associated with improvement in choice but not simple reaction time. This result suggests that simple reaction time is not exclusively dopamine dependent. Starkstein et $a l^{31}$ studied six patients with a mean illness duration of 11.6 years who showed marked motor fluctuations, including dyskinesia. SRT did not change from the "off" to the "on" state although movement time improved significantly. This study provides further evidence of a dissociation between SRT and clinical motor disability as measured by movement time and suggests that simple reaction time is not mediated through dopamine.

The studies to date have not compared reaction time in untreated and treated groups and have not directly addressed the effect of initiation of treatment in early, untreated PD. A recent study, however, has shown involvement of dopamine in motor readiness in rats with unilateral dopamine depletion induced by hydroxydopamine injections into the striatum. ${ }^{32}$ Unlike the patients of our study, these animals showed a failure to improve SRT performance as a function of interval between warning signal and imperative stimulus. However, 6-hydroxydopamine produces profound dopamine depletion within the striatum; our patients, as a group, had a short disease duration and relatively mild disease (table 1). Thus the discrepancy between our study and that of Brown and Robbins may be due to differences in the extent of dopamine depletion within the striatum. Our results do not exclude an effect of dopamine on reaction time in more severe PD; nevertheless, the observation that dopamine depletion sufficient to cause clinical signs of PD is not necessarily accompanied by deficits in motor readiness suggests that motor readiness is dependent on very low levels of dopaminergic neurotransmission or, perhaps more likely, involves non-dopaminergic as well as dopaminergic systems. On-off swings are associated with change in affect-arousal ${ }^{33}$ which may be dependent on noradrenergic activity. ${ }^{34}$ Reaction time and vigilance are related to CSF levels of the noradrenaline metabolite 3 methoxy-4-hydroxyphenylethylene glycol (MHPG). ${ }^{35}$ Thus reaction time may be partially dependent on the integrity of noradrenergic pathways whereas movement 
time is related principally to dopaminergic activity.

In conclusion our study showed that early untreated patients with PD show prolongation of SRT which is not due to deficits in attentional focussing, stimulus anticipation or temporal predictability. The deficits correlate with cognitive and motor measures but do not improve on treatment, despite clinical benefit. The results suggest that PD patients show slowing in processes concerned with orientation of both cognitive and motor responses to a stimulus. The deficits are not exclusively related to nigrostriatal dopamine deficiency.

This research was supported by grants from Roche Products, UK and the Parkinson's Disease Society, UK. We thank Drs GAB Davies-Jones, J Gumpert and G Venables for referring patients for study, Dr $N$ Harvey for provision of some of the patients for study, Dr N Harvey for provision of some of the
Beck scores on the PD group and Dr E Sullivan for useful discussion.

1 Brown RG, Marsden CD. Neuropsychology and cognitive function in Parkinson's disease: an overview. In: Marsden
$\mathrm{CD}$, Fahn S, eds. Movement disorders 2. London: ButterCD, Fahn S, eds. Move
worth 1987:99-123.

2 Sagar HJ, Sullivan EV. Patterns of cognitive impairment in dementia. In: Kennard C, ed. Recent advances in clinical
neurology, Vol 5. Edinburgh: Churchill Livingstone, neurology, Vol

3 Benecke R, Rothwell JC, Dick JPR, Day BL, Marsden CD. Disturbance of sequential movements in patients with Parkinson's disease. Brain 1987;110:361-79.

4 Robertson C, Flowers KA. Motor set in Parkinson's disease. I Neurol Neurosurg Psychiatry 1990;53:583-92.

5 Benecke R, Rothwell JC, Dick JPR, Day BL, Marsden CD. Performance of simultaneous movements in patients with Parkinson's disease. Brain 1986;109:739-57.

6 St-Cyr JA, Taylor AE, Lang AE. Procedural learning and neostriatal dysfunction in man. Brain 1988;111:941-59.

7 Rabbit PMA, Vyas S. Age and the rate of preparation for signals and for responses. Exper Brain Res signals and $1982 ; 5: 216-22$.

8 Evarts EV, Teravainen H, Calne DB. Reaction time in Parkinson's disease. Brain 1981;104:167-83.

9 Bloxham CA, Dick DJ, Moore M. Reaction times and attention in Parkinson's disease. $\mathcal{f}$ Neurol Neurosur Psychiatry 1987;50:1178-83.

10 Goodrich S, Henderson L, Kennard C. On the existence of an attention-demanding process peculiar to simple reaction time: Converging evidence from Parkinson's disease. Cognitive Neuropsychology 1989;6(3):309-31.

11 Brown RG, Marsden CD. Visuospatial function in Parkinson's disease. Brain 1986;109:987-1002.

12 Pullman SL, Watts RL, Juncas JL, Chase TN, Sanes JN. Dopaminergic effects on simple and choice reaction time performance in Parkinson's disease. Neurology performance

13 Blessed G, Tomlinson BE, Roth $M$. The association between quantitative measures of dementia and of senile changes in central grey matter of elderly subjects. Br $\mathcal{F}$ Psychiatry
1968;114:797-811

14 Nelson HE, O'Connell A. Dementia: the estimation of premorbid intelligence level using the New Adult Reading Test. Cortex 1978;14:234-46.

15 Wechsler D, Stone CP. Wechsler Memory Scale. New York: Psychological Corporation, 1945.

16 Milner B. Effects of different brain lesions on card sorting: the role of the frontal lobes. Res Publ Asst Res Nerve Ment Dis 1958;36:244-57.

17 Beck AT, Ward CH, Mendelson M, Mock J, Erbaugh J. An inventory for measuring depression. Arch Gen Psychiatry 1961;4:561-71.

18 Brown RG, Marsden CD, Quinn N, et al. Alterations in affect-arousal state during fluctuations in motor function in Parkinson's disease. I Neurol Neurosurg Psychiatry 1984;47:454-65.

19 Sheridan MR, Flowers KA, Harwell J. Programming and execution of movement in Parkinson's disease. Brain execution of movem

20 Stelmach GE, Worringham CJ, Strand EA. Movement preparation in Parkinson's disease. Brain

21 Rafal RD, Posner MI, Walker JA, Friedrick FJ. Cognition and the basal ganglia. Separating the mental and motor components of performance in Parkinson's disease. Brain 1984;107:1083-94.

22 Sagar HJ, Jordan N, Cooper J, Sullivan EV. The dissociation between cognition and motor control in Parkinson's disease. Neurology (Sup 1) 1990;40:168.

23 Bloxham CA, Mindel TA, Frith CD. Initiation and execution of predictable and unpredictable movements in Parkinson's disease. Brain 1984;107:371-84.

24 Marsden CD. The mysterious motor function of the basal ganglia: The Robert Wartenberg lecture. Neurology 1982;32:514-39.

25 Frith CD Done DJ. Routes to action in reaction time tasks. Psychol Res 1984;48:169-77.

26 Goldberg G. Supplementary motor area structure and function: review and hypotheses. Behav Brain $\mathrm{Sci}$ 1985;8:567-88.

27 Dick JPR, Rothwell JC, Day BL, et al. The Bereitschaftspotential is abnormal in Parkinson's disease. Brain 1989;112:233-44

28 Deiber M-P, Passingham RE, Colebatch JG, Friston KJ, Nixon PD, Frackowiak RSJ. Cortical areas and the selection of movement: a study with positron emission tomography. Exp Brain Res 1991;84:393-402.

29 Marsden CD. Slowness of movement in Parkinson's disease. In: Fahn S, Marsden CD, eds. Movement disorders, 4 (suppl 1). New York: Raven Press, 1989:26-37.

30 Parks RW, Loewenstein DA, Dodrill KL, et al. Cerebral metabolic effects of a verbal fluency test: a PET scan metabolic effects of a verbal fluency test: a PET

31 Starkstein SE, Esteguy M, Berthier ML, Garcia H, Leiguarda $R$. Evoked potentials, reaction time and cognitive guarda $\mathrm{R}$. Evoked potentials, reaction time and cognitive performance in on and off phases of Parkinson's
$\Im$ Neurol Neurosurg Psychiatry 1989;52:338-40.

32 Brown VJ, Robbins TW. Simple and choice reaction time performance following unilateral striatal dopamine depletion in the rat. Impaired motor readiness but preserved response preparation. Brain 1991;114:513-25.

33 Brown RG, Marsden CD, Quinn N, Wyke MA. Alterations in cognitive performance and affect-arousal state during fluctuations in motor function in Parkinson's disease. Neurol Neurosurg Psychiatry 1984;47:454-65.

34 Marsden CD, Jenner P. The mode of action of 1-dopa in Parkinson's disease. Trends in Neurosciences 1981;4:148-50.

35 Stern Y, Mayeux R, Cote L Reaction times and attention in Parkinson's disease. Arch of Neurol 1984;41:1086-9. 